\title{
Nitrergic nerves derived from the pterygopalatine ganglion innervate arteries irrigating the cerebrum but not the cerebellum and brain stem in monkeys
}

\author{
Kazuhide Ayajiki $^{1}$, Shuhei Kobuchi ${ }^{1}$, Masashi Tawa ${ }^{2}$ and Tomio Okamura ${ }^{2}$
}

The functional roles of the nitrergic nerves innervating the monkey cerebral artery were evaluated in a tension-response study examining isolated arteries in vitro and cerebral angiography in vivo. Nicotine produced relaxation of arteries by stimulation of nerve terminals innervating isolated monkey arteries irrigating the cerebrum, cerebellum and brain stem. Relaxation of arteries induced by nicotine was abolished by treatment with $\boldsymbol{N}^{\mathrm{G}}$-nitro-L-arginine, a nitric oxide synthase inhibitor, and was restored by addition of L-arginine. Cerebral angiography showed that electrical stimulation of the unilateral greater petrosal nerve, which connects to the pterygopalatine ganglion via the parasympathetic ganglion synapse, produced vasodilatation of the anterior, middle and posterior cerebral arteries in the stimulated side. However, stimulation failed to produce vasodilatation of the superior and anterior-inferior cerebellar arteries and the basilar artery in anesthetized monkeys. Therefore, nitrergic nerves derived from the pterygopalatine ganglion appear to regulate cerebral vasomotor function. In contrast, circulation in the cerebellum and brain stem might be regulated by nitrergic nerves originating not from the pterygopalatine ganglion, but rather from an unknown ganglion (or ganglia).

Hypertension Research (2012) 35, 88-92; doi:10.1038/hr.2011.163; published online 6 October 2011

Keywords: angiography; monkey cerebral artery; nerve stimulation; nitrergic nerve; relaxation

\section{INTRODUCTION}

It has been reported that nitric oxide (NO) acts as a neurotransmitter in the vasodilator nerves innervating the cerebral arteries in several mammals. ${ }^{1,2}$ Histochemistry has showed that nitric oxide synthase (NOS)-containing nerves originating from the pterygoparatine ganglion (PPG) innervate arteries irrigating the cerebrum in many species. ${ }^{3-6}$ We have reported that electrical stimulation of the unilateral PPG or the greater petrosal nerve (GPN), a preganglionic parasympathetic nerve that synaptically connects to the PPG, dilates the ipsilateral anterior cerebral artery (ACA), and the middle cerebral artery (MCA) in anesthetized monkeys ${ }^{7}$ and dogs. ${ }^{8}$ We have also found that these dilatations are suppressed by a NOS inhibitor. Furthermore, electrical stimulation of unilateral nerve bundles containing parasympathetic nerves produced an increase of cerebral blood flow in the ipsilateral brain of rats treated with atropine and capsaicin; this increase of cerebral blood flow in the temporo-parietal area was abolished by a NOS inhibitor. ${ }^{9}$ However, it remains unclear whether or not nitrergic nerves from the PPG innervate other major arteries irrigating the cerebrum, cerebellum and brain stem, including the posterior cerebral artery (PCA), superior cerebellar artery (SCA), anterior inferior cerebellar artery (AICA) and basilar artery (BA).
In this study, cerebral angiography was performed to measure the diameters of arteries irrigating the cerebrum, cerebellum and brain stem when electrical stimulation of the GPN was performed in anesthetized Japanese monkeys. Furthermore, a tension-response study was performed to determine whether or not the arteries that irrigate the posterior cerebrum, cerebellum and brain stem, when isolated from the Japanese monkey brain, showed comparable responses to those observed in cerebral angiography studies.

\section{METHODS}

The Animal Care and Use Committee at Shiga University of Medical Science approved the use of monkey blood vessels in this study.

\section{In vivo study}

Six Japanese monkeys (Macaca fuscata; both male and female), approximately $10-12$ years of age and weighing $8-10 \mathrm{~kg}$, were premedicated with intramuscular injections of $15 \mathrm{mg} \mathrm{kg}^{-1}$ ketamine and anesthetized intravenously with $20 \mathrm{mg} \mathrm{kg}^{-1}$ sodium pentobarbital. Stable anesthetic conditions were maintained by additional injections of pentobarbital as needed. Atropine $\left(1 \mathrm{mg} \mathrm{kg}^{-1}\right)$ was intravenously injected to inhibit the parasympathetic reflex and pentazocine $\left(10 \mathrm{mg} \mathrm{kg}^{-1}\right)$ was subcutaneously injected to abolish pain, which is resistant to pentobarbital anesthesia. Monkeys were intubated when necessary, but were usually permitted to breathe spontaneously. $\mathrm{PO}_{2}$ and $\mathrm{PCO}_{2}$

${ }^{1}$ Department of Pharmacy, School of Pharmacy, Hyogo University of Health Sciences, Chuo-ku, Kobe, Japan and ${ }^{2}$ Department of Pharmacology, Shiga University of Medical Science, Seta, Otsu, Japan

Correspondence: Dr K Ayajiki, Department of Pharmacy, School of Pharmacy, Hyogo University of Health Sciences, Chuo-ku, Kobe 650-8530, Japan.

E-mail: ayajiki@huhs.ac.jp

Received 5 January 2011; revised 7 June 2011; accepted 23 June 2011; published online 6 October 2011 
were stable during the experiment. Arterial systolic and diastolic pressures were monitored by a pressure transducer (NEC San-ei, Tokyo, Japan) by a catheter inserted into the left femoral artery. The monkeys' body temperature was kept at $37^{\circ} \mathrm{C}$ on a heated operating table. To reach the GPN originating from the superior salivatory nucleus, ${ }^{7}$ a post-auricular incision was made and the external auditory canal was cut and retracted anteriorly. After the temporal bone was removed with a cutting burr, the facial nerve was cut at the stylomastoid foramen and exposed along its course from the foramen to the GPN. With the aid of a surgical microscope, a fine bipolar concentric stimulating electrode was inserted into the GPN and then fixed with dental cement. The nerve was stimulated with electrical pulses $(1 \mathrm{~ms}$ in duration and at a frequency of $10 \mathrm{~Hz}$, with an intensity of $10 \mathrm{~V}$ for $20 \mathrm{~s}$ ); $5 \mathrm{~s}$ after the start of stimulation, the contrast medium for angiography was injected. Transfemoral internal carotid or vertebrobasilar angiography was performed with a digital subtraction angiography system (DFA-3-30, Hitachi Medical, Tokyo, Japan) at the same magnification throughout the experiment. Internal carotid angiography enhances ACA and MCA, and vertebrobasilar angiography enhances PCA, $\mathrm{BA}, \mathrm{SCA}$ and AICA. The contrast medium iopamidol (Iopamiron, $2 \mathrm{ml}$, Schering, Germany) was injected by an auto-injector (Angiomat 6000, Liebel-Flarsheim, Co., Cincinnati, OH, USA) connected to the angiography system in each angiography session. Data were stored on a digital data recorder (Hitachi Medical). The diameters of the arteries (ACA, MCA, PCA, BA, SCA and AICA) were measured at two selected points with an image analyzer included with the angiography system. The two values were averaged and the results were expressed as a percentage of the control artery diameter obtained just before the electrical stimulation, as described in our previous studies. ${ }^{7,10}$ Arterial diameters before, during and $10 \mathrm{~min}$ after the nerve stimulation were measured by angiography.

\section{In vitro study}

Five Japanese monkeys (Macaca fuscata; both male and female), approximately $10-12$ years of age and weighing $8-10 \mathrm{~kg}$, were killed by exsanguination from the common carotid artery under deep anesthesia with an intramuscular injection of ketamine $\left(40 \mathrm{mg} \mathrm{kg}^{-1}\right)$ and an intravenous injection of sodium pentobarbital $\left(30 \mathrm{mg} \mathrm{kg}^{-1}\right)$. The brain was rapidly removed, and the ACA (0.8-0.9 mm outside diameter), MCA (1.0-1.2 mm), PCA $(0.7-0.8 \mathrm{~mm})$, BA $(0.9-1.1 \mathrm{~mm})$, SCA $(0.5-0.6 \mathrm{~mm})$ and AICA $(0.3-0.4 \mathrm{~mm})$ were isolated as shown in Figure 1. The arteries were cleaned of surrounding tissue and were cut into helical strips approximately $10 \mathrm{~mm}$ in length. The endothelium of all strips was removed by gently rubbing the intimal surface with a cotton ball. The strips were vertically fixed between hooks in a muscle bath $(10 \mathrm{ml}$ capacity) containing a modified Ringer-Locke solution that was kept at $37 \pm 0.3^{\circ} \mathrm{C}$ and aerated with a mixture of $95 \% \mathrm{O}_{2}-5 \% \mathrm{CO}_{2}$. The hook anchoring the upper end of the strips was connected to the lever of a force-displacement transducer (NihonKohden Kogyo Co., Tokyo, Japan). The resting tension was adjusted to $1.0 \mathrm{~g}$, which was optimal for inducing a maximal contraction. The constituents of the Ringer-Locke solution were (in $\mathrm{mM}$ ) as follows: $120 \mathrm{NaCl}, 5.4 \mathrm{KCl}, 2.2 \mathrm{CaCl}_{2}$, $1.0 \mathrm{MgCl}_{2}, 25.0 \mathrm{NaHCO}_{3}$ and 5.6 dextrose. The $\mathrm{pH}$ of the solution was 7.367.43. Before the start of the experiments, all of the strips were allowed to equilibrate for 60-90 $\mathrm{min}$ in the bathing media, during which the fluid was replaced every $10-15 \mathrm{~min}$.

Isometric contractions and relaxations were displayed on a pen recorder. The contractile response to $30 \mathrm{~mm} \mathrm{~K} \mathrm{~K}^{+}$was obtained first, and the arterial strips were repeatedly washed with fresh media and equilibrated. The strips were partially contracted with prostaglandin (PG) $\mathrm{F}_{2 \alpha}\left(0.2-1 \times 10^{-6} \mathrm{M}\right)$, the contraction being $35-40 \%$ of the magnitude of the contraction induced by $30 \mathrm{mM} \mathrm{K} \mathrm{K}^{+}$. A single concentration $\left(10^{-4} \mathrm{M}\right)$ of nicotine was directly added to the bathing media. The initial responses to nicotine were compared in the absence or presence of a NOS inhibitor ( $N^{\mathrm{G}}$-nitro-L-arginine (L-NA), $10^{-5} \mathrm{M}$ ) and/or L-arginine $\left(3 \times 10^{-4} \mathrm{M}\right)$. At the end of each series of experiments, papaverine $\left(10^{-4} \mathrm{M}\right)$ was applied to attain maximum relaxation. Relaxations induced by nicotine were expressed as a percentage of those induced by papaverine.

\section{Statistics and drugs used}

The results shown in the figures are expressed as mean values \pm s.e.m. Statistical analyses were made using the Student's unpaired $t$-test for two groups or the Tukey's test following one-way analysis of variance for three groups. The drugs used were prostaglandin (PG) $\mathrm{F}_{2 \alpha}$ (Pfizer Inc, Tokyo, Japan), L-arginine, nicotine (base), L-NA (Peptide Institute, Minoh, Japan), papaverine hydrochloride, pentobarbital sodium (Nacalai Tesque, Kyoto, Japan), atropine sulfate (Mitsubishi Tanabe Pharma, Osaka, Japan), pentazocine (Astellas Pharma, Tokyo, Japan) and ketamine (Daiichi Sankyo, Tokyo, Japan).

\section{RESULTS}

\section{In vivo study}

Carotid or vertebrobasilar angiography in anesthetized monkeys showed that electrical stimulation of the right GPN at a frequency of $10 \mathrm{~Hz}$ for $20 \mathrm{~s}$ dilated the ipsilateral ACA, MCA and PCA, which irrigate the cerebrum. However, this stimulation did not dilate the distal and proximal BA, SCA and AICA, which irrigate the cerebellum and brain stem (Figure 2). Typical vertebrobasilar angiography showing GPN stimulation-induced arterial dilatation of the PCA, but not of the SCA, AICA and BA, is presented in Figure 3.

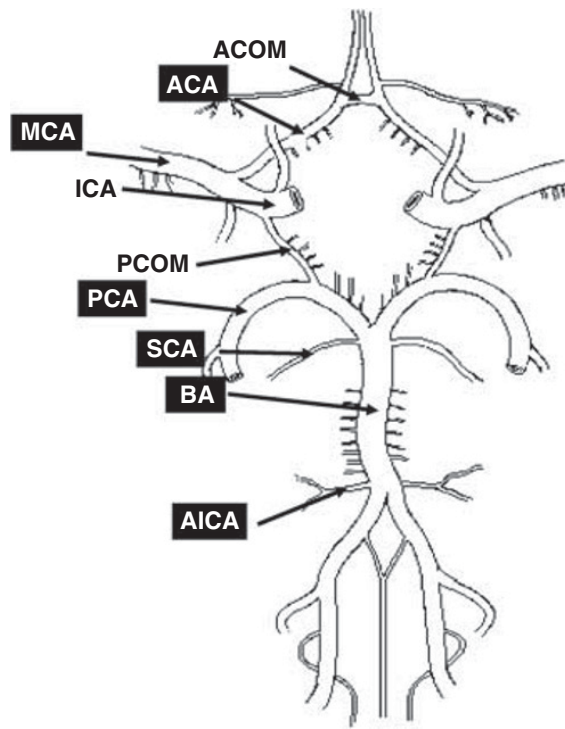

ACOM, Anterior Communicating Artery
ACA, Anterior Cerebral Artery
ICA, Internal Carotid Artery
MCA, Middle Cerebral Artery
PCOM, Posterior Communicating Artery
PCA, Posterior Cerebral Artery
SCA, Superior Cerebellar Artery
BA, Basilar Artery
AICA, Anterior Inferior Cerebellar Artery
, used in the present study

Figure 1 Illustration of monkey arteries irrigating the cerebrum, cerebellum and brainstem. Arrows point to the arteries. White words in black squares identify the portions of arteries that were used in this study. Abbreviations are identified on the right side of the figure. 


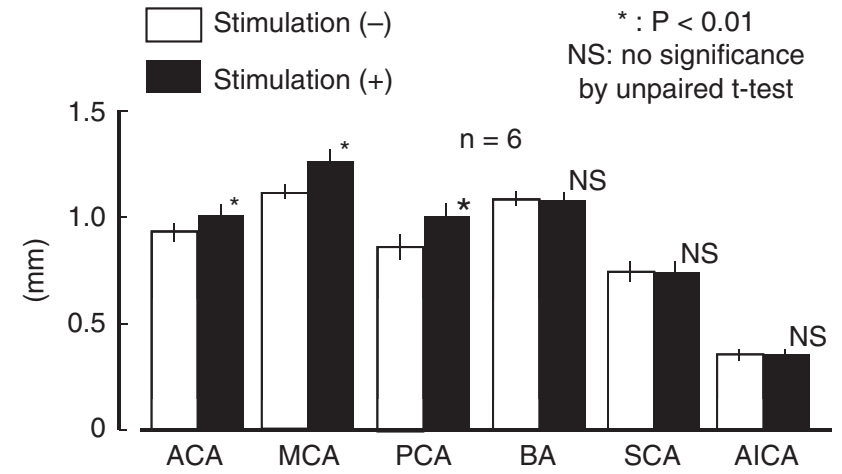

Figure 2 Comparison of changes in diameters of the monkey ACA, MCA, PCA, BA, SCA and AICA in the non-stimulated (open squares) and stimulated (closed squares) conditions. Stimulation of the ipsilateral petrosal nerve in anesthetized monkeys $(n=6)$ was delivered with electrical pulses ( $1 \mathrm{~ms}$ duration, frequency of $10 \mathrm{~Hz}$ and $10 \mathrm{~V}$ intensity for $20 \mathrm{~s}$ ). The ordinate denotes the diameter increase $(\mathrm{mm})$ in response to nerve stimulation. Vertical bars represent s.e.m. Significantly different from the value in nonstimulated condition, ${ }^{*} P<0.01$; significantly different from the value with the stimulation (unpaired $t$-test). NS, no significance.

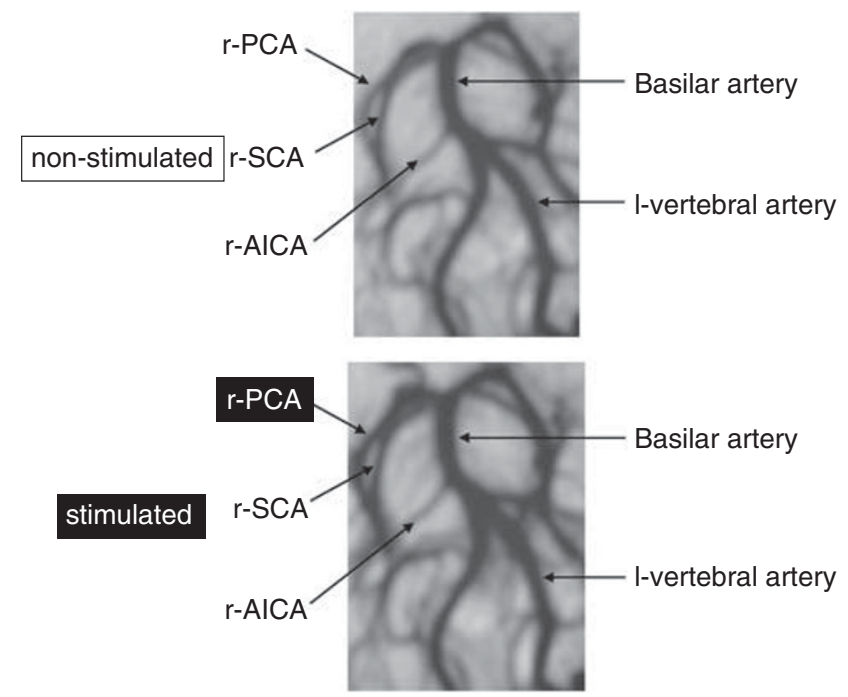

Figure 3 Typical angiography showing changes in the diameter of the PCA, SCA, BA, AICA and vertebral arteries by electrical stimulation of the petrosal nerve ( $1 \mathrm{~ms}$ duration, frequency of $10 \mathrm{~Hz}$ and $10 \mathrm{~V}$ intensity for $20 \mathrm{~s}$ ) before (upper, non-stimulated) and during (lower, stimulated) nerve stimulation in an anesthetized monkey.

\section{In vitro study}

The monkeys used for the in vivo study were given additional injections of thiopental and then killed by bleeding after the experiment was finished. The brain (cerebrum, cerebellum and brain stem) from the non-operated side was stored overnight for this study. In helical strips of the ACA, MCA, PCA, SCA, AICA and BA denuded of the endothelium and partially contracted with $\mathrm{PGF}_{2 \alpha}$, nicotine $\left(10^{-4} \mathrm{M}\right)$, which stimulates nerve terminals innervating arteries, produced reproducible relaxations. These relaxations were abolished by treatment with L-NA $\left(10^{-6} \mathrm{M}\right)$, as shown in Figure 4. Additional treatment with $\mathrm{L}$-arginine $\left(3 \times 10^{-4} \mathrm{M}\right)$ partially restored the relaxation induced by nicotine.

\section{DISCUSSION}

This study revealed that in anesthetized monkeys, electrical stimulation of the unilateral GPN dilated the ipsilateral ACA and MCA, as reported; ${ }^{7}$ this stimulation also dilated the ipsilateral PCA, but not the SCA, AICA or BA. Our previous study ${ }^{4}$ showed the presence of abundant nerve cells, bundles and fibers containing NOS immunoreactivity in the monkey PPG. We have reported that nicotine produces relaxations that chemically stimulate perivascular nerve terminals to liberate neurotransmitters. ${ }^{11-13}$ We isolated arteries irrigating the cerebrum, cerebellum and brain stem from monkeys; these arteries responded to chemical nerve stimulation by application of nicotine with relaxations that were abolished by treatment with L-NA. The effect of L-NA was partially reversed by the addition of L-arginine. Similar results with electrical or chemical stimulation have also been obtained in canine, bovine, porcine and human arteries irrigating the cerebrum, cerebellum and brain stem. ${ }^{14-18}$ In addition, we previously reported that release of NO from isolated, superfused canine arteries, irrigating the cerebrum with superfusate in response to transmural electrical nerve stimulation or in response to chemical nerve stimulation with nicotine, ${ }^{11}$ was abolished by treatment with NOS inhibitors and tetrodotoxin (for electrical stimulation) or hexamethonium (for nicotine). The same was true of the nicotine-induced increase in tissue cyclic guanosine monophosphate content. These findings support the hypothesis that NO has a crucial role as a neurotransmitter in the vasodilator nerves innervating arteries of various mammals. ${ }^{19}$ In the ipsilateral ACA and MCA isolated from dogs in which the unilateral PPG is degenerated, the relaxant response to nicotine is abolished, whereas the response is unaffected in the contralateral ACA and MCA. These results suggest that the nitrergic vasodilator innervations of the ACA and MCA derive from the ipsilateral PPG in dogs. ${ }^{20}$

Unilateral denervation of the monkey PPG decreased the diameter of the ipsilateral ACA and MCA. ${ }^{7}$ We previously reported that intracisternal injections of L-NA constrict the BA and that this effect is reversed by L-arginine. Furthermore, the vasoconstrictor action of L-NA is significantly attenuated by treatment with hexamethonium, suggesting that neurogenic NO, which is continuously released under resting conditions, is involved in basilar arterial dilatation in anesthetized monkeys and dogs. ${ }^{21,22}$ These findings strongly suggest that nitrergic tonic discharges from the vasomotor center contribute to the maintenance of cerebro-arterial dilatation and decreased arterial resistance.

In in vivo studies measuring the diameter of rat, mouse, goat and pig pial arteries, and arterioles or cerebral vascular resistance, intravenous injection or topical application of NOS inhibitors produces vasoconstriction or decreases blood flow. ${ }^{23-27}$ It is postulated that vasoconstriction is partially mediated by the suppression of $\mathrm{NO}$, synthesized from L-arginine in the perivascular nerve terminals innervating the arteries that irrigate the cerebrum, as treatment with a ganglion blocker can suppress vasoconstriction or cause a decrease in blood flow. ${ }^{21}$ We therefore conclude that in the arteries irrigating the cerebrum, cerebellum and brain stem, constrictions induced by intravenous L-NA may be partially associated with the suppression of nitrergic function.

In the monkey, stimulation of the GPN also dilated the ipsilateral ACA and MCA to a similar extent, as did stimulation of the PPG. Responses to GPN stimulation, but not to PPG stimulation, were abolished by hexamethonium. In contrast, responses to stimulation of the nerve and the ganglion were suppressed by L-NA. ${ }^{7}$

Using a combination of Fluoro-Gold (Fluorochrome, LLC, Denver, CO, USA) retrograde tracing and immunohistochemistry, Kadota 

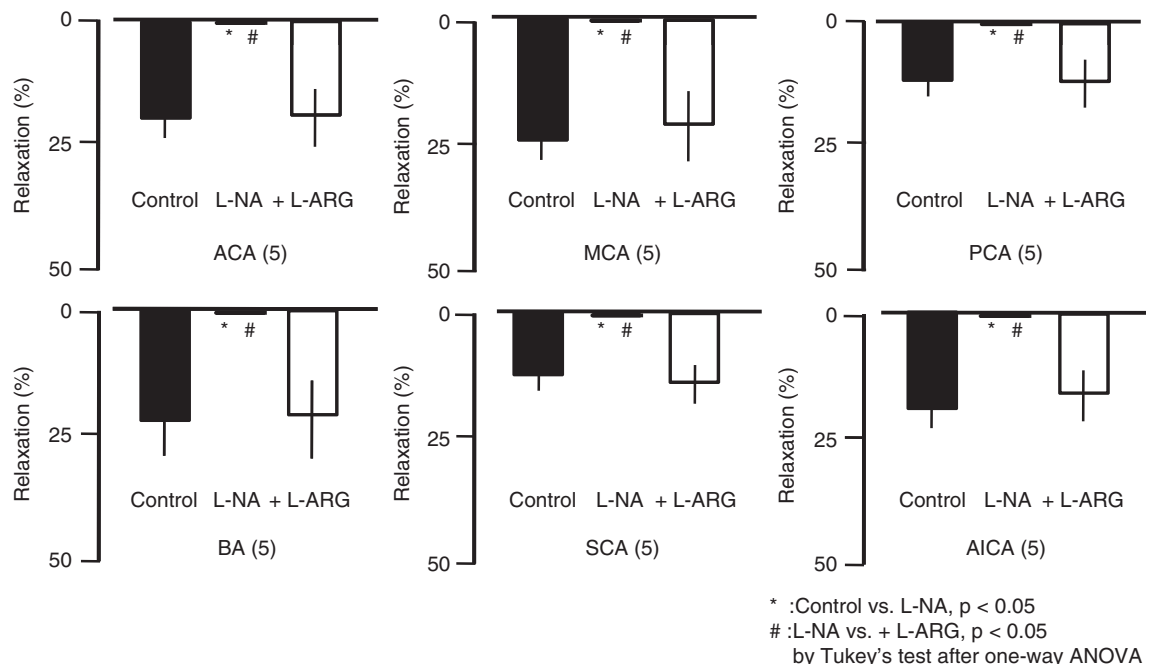

Figure 4 Modification by L-NA $\left(10^{-6} \mathrm{M}\right)$ and L-NA plus L-arginine $\left(+\mathrm{L}-\mathrm{ARG}, 3 \times 10^{-4} \mathrm{M}\right)$ of the relaxation induced by nicotine $\left(10^{-4} \mathrm{M}\right)$ in the isolated monkey ACA, MCA, PCA, BA, SCA and AICA ( $n=5$ each). The ordinate denotes the relaxation to nicotine. Vertical bars represent s.e.m. ${ }^{*} P<0.05$; significantly different from the control value vs. L-NA treatment. ${ }^{\#} P<0.05$; significantly different from the value under treatment with L-NA vs. that with L-NA plus L-arginine (Tukey's test after one-way analysis of variance).

et al. ${ }^{28}$ reported that the origins of NOS-containing nerve fibers in the rat BA derive from the sphenopalatine (pterygopalatine, PPG), otic, trigeminal, nodose ganglia and spinal ganglia. These results indicate that NOS-containing nerve fibers in the rat BA have multiple origins. Keller et al. ${ }^{29}$ also reported that the nerves innervating the BA in the cat have multiple origins. However, it is known that the trigeminal and superior vagal ganglia mainly send sensory nerves from the BA to the central nervous system. In addition, superior cervical and stellate ganglion mainly send sympathetic nerves, not including nitrergic nerves, to the BA. $^{29}$ Furthermore, we have observed that L-NA, when applied around the BA with a fine needle through the foramen magnum, constricted the $\mathrm{BA}$ together with arteries irrigating the cerebrum. ${ }^{30}$ These results indicate that L-NA could not localize to the BA and that it was possibly circulated by cerebrospinal fluid in the monkey brain. This observation suggests the difficulty of conducting a retrograde tracing study in the monkey. Moreover, we have performed surgeries in order to stimulate the otic ganglion, a possible origin of the nitrergic nerve that innervates the basilar and/or cerebellar arteries. However, we failed to stimulate the ganglion satisfactorily. Therefore, the origin of the nitrergic nerve innervating the arteries that irrigate the monkey cerebellum and brain stem remains to be clarified.

In conclusion, nitrergic nerves derived from the PPG appear to regulate the vasomotor function of arteries irrigating the cerebrum, whereas nitrergic nerves from a ganglion other than the PPG may regulate circulation in the cerebellum and brain stem.

\section{CONFLICT OF INTEREST}

The authors declare no conflict of interest.

1 Toda N, Okamura T. The pharmacology of nitric oxide in the peripheral nervous system of blood vessels. Pharmacol Rev 2003; 55: 271-324.

2 Lee TJ. Sympathetic modulation of nitrergic neurogenic vasodilation in cerebral arteries. Jpn J Pharmacol 2002; 88: 26-31.

3 Nozaki K, Moskowitz MA, Maynard KI, Koketsu N, Dawson TM, Bredt DS, Snyder SH Possible origins and distribution of immunoreactive nitric oxide synthase-containing nerve fibers in cerebral arteries. J Cereb Blood Flow Metab 1993; 13: 70-79.
4 Yoshida K, Okamura T, Toda N. Histological and functional studies on the nitroxidergic nerve innervating monkey cerebral, mesenteric and temporal arteries. Jpn J Pharmacol 1994; 65: 351-359.

5 Toda N, Ayajiki K, Okamura T. Cerebral blood flow regulation by nitric oxide: recent advances. Pharmacol Rev 2009; 6: 62-97.

6 Talman WT, Corr J, Nitschke Dragon D, Wang D. Parasympathetic stimulation elicits cerebral vasodilatation in rat. Auton Neurosci 2007; 133: 153-157.

7 Toda N, Tanaka T, Ayajiki K, Okamura T. Cerebral vasodilatation induced by stimulation of the pterygopalatine ganglion and greater petrosal nerve in anesthetized monkeys. Neuroscience 2000; 96: 393-398.

8 Toda N, Ayajiki K, Tanaka T, Okamura T. Preganglionic and postganglionic neurons responsible for cerebral vasodilation mediated by nitric oxide in anesthetized dogs. J Cereb Blood Flow Metab 2000; 20: 700-708.

9 Ayajiki K, Fujioka H, Shinozaki K, Okamura T. Effects of capsaicin and nitric oxide synthase inhibitor on increase in cerebral blood flow induced by sensory and parasympathetic nerve stimulation in the rat. J Appl Physiol 2005; 98: 1792-1798.

10 Ayajiki K, Tanaka T, Okamura T, Toda N. Evidence for nitroxidergic innervation in monkey ophthalmic arteries in vivo and in vitro. Am J Physiol Heart Circ Physiol 2000; 279: H2OO6-H2O12.

11 Toda N. Relaxant responses to transmural stimulation and nicotine of dog and monkey cerebral arteries. Am J Physiol 1982; 243: H145-H153.

12 Nedergaard OA. Effects of nicotine on neuroeffector transmission in blood vessels. In: Rand M, and Thurau KL. (eds). The Pharmacology of Nicotine. IRL: Washington, DC, 1988 pp. 143-162.

13 Toda N, Okamura T. Possible role of nitric oxide in transmitting information from vasodilator nerve to cerebroarterial muscle. Biochem Biophys Res Commun 1990; 170: 308-313.

14 Toda N, Okamura T. Modification by L-NG-monomethyl arginine (L-NMMA) of the response to nerve stimulation in isolated dog mesenteric and cerebral arteries. Jpn J Pharmacol 1990; 52: 170-173.

15 Toda N, Okamura T. Mechanism underlying the response to vasodilator nerve stimulation in isolated dog and monkey cerebral arteries. Am J Physiol Heart Circ Physiol 1990; 259: H1511-H1517.

16 Ayajiki K, Okamura T, Toda N. Nitric oxide mediates, and acetylcholine modulates, neurally induced relaxation of bovine cerebral arteries. Neuroscience 1993; 54: $819-825$.

17 Lee TJ, Sarwinski SJ. Nitric oxidergic neurogenic vasodilation in the porcine basilar artery. Blood Vessels 1991; 28: 407-412.

18 Toda N. Mediation by nitric oxide of neurally-induced human cerebral artery relaxation. Experientia 1993; 49: 51-53.

19 Toda N, Okamura T. Nitroxidergic nerve: regulation of vascular tone and blood flow in the brain. J Hypertens 1996; 14: 423-434.

20 Toda N, Ayajiki K, Yoshida K, Kimura H, Okamura T. Impairment of pterygopalatine ganglion of nitroxidergic vasodilator nerve function in canine cerebral and retinal arteries. Circ Res 1993; 72: 206-213.

21 Okamura T, Ayajiki K, Toda N. Basilar arterial construction caused by intracisternal $\mathrm{N}^{\mathrm{G}}$-nitro-L-arginine in anesthetized monkeys. Cardiovasc Res 1995; 30: 663-667.

22 Toda N, Ayajiki K, Okamura T. Neural mechanism underlying basilar arterial constriction by intracisternal L-NNA in anesthetized dogs. Am J Physiol 1993; 265: H103-H107. 
23 Armstead WM, Zuckerman SL, Shibata M, Parfenova H, Leffler CW. Different pial arteriolar responses to acetylcholine in the newborn and juvenile pig. $J$ Cereb Blood Flow Metab 1994; 14: 1088-1095.

24 Faraci FM. Role of nitric oxide in regulation of basilar artery tone in vivo. Am J Physiol 1990; 259: H1216-H1221.

25 Faraci FM. Role of endothelium-derived relaxing factor in cerebral circulation: large arteries vs. microcirculation. Am J Physiol 1991; 261: H1038-H1042.

26 Fernández N, García JL, García-Villalón AL, Monge L, Gómez B, Diéguez G. Cerebral blood flow and cerebrovascular reactivity after inhibition of nitric oxide synthesis in conscious goats. Br J Pharmacol 1993; 110: 428-434.
27 Rosenblum WI, Nishimura H, Nelson GH. Endothelium-dependent L-Arg- and LNMMA-sensitive mechanisms regulate tone of brain microvessels. Am J Physiol 1990; 259: H1396-H1401.

28 Kadota O, Matsuda S, Ohta S, Kumon Y, Sakaki S, Sakanaka M. Origins of nitric oxide synthase-containing nerve fibers in the rat basilar artery with reference to the fine structure of the nerve fibers. Brain Res 1996; 706: 129-136.

29 Keller JT, Beduk A, Saunders MC. Origin of fibers innervating the basilar artery of the cat. Neurosci Lett 1985; 58: 263-268.

30 Toda N, Okamura T. Role of nitric oxide in neurally induced cerebroarterial relaxation. J Pharmacol Exp Ther 1991; 258: 1027-1032. 